\title{
Increased a-Actinin-2 Expression in the Atrial Myocardium of Patients with Atrial Fibrillation Related to Rheumatic Heart Disease
}

\author{
Lei Zhang Nan Zhang Xuejiao Tang Fajin Liu Suxin Luo Hua Xiao \\ Department of Cardiology, The First Affiliated Hospital of Chongqing Medical University, Chongqing, China
}

\author{
Key Words \\ Atrial fibrillation - Transforming growth factor $-\beta_{1} / \mathrm{Smad}$ \\ signaling pathway $\cdot$ Cytoskeleton $\cdot a-A c t i n i n-2 \cdot$ Atrial \\ structural remodeling
}

\begin{abstract}
Objectives: Atrial fibrosis, a marker of atrial structural remodeling, plays a critical role in atrial fibrillation (AF). aActinin-2 is associated with structural remodeling related to stretching. The transforming growth factor- $\beta_{1}$ (TGF- $\beta 1$ )/ Smad pathway plays an important role in atrial fibrosis. We investigated the effects of the TGF- $\beta 1 /$ Smad signaling pathway on a-actinin-2 in atrial fibrosis in patients with AF. Methods: Forty-one right atrial specimens obtained from patients with rheumatic heart disease (RHD) were divided into a chronic (c)AF group, i.e. RHD + CAF $(n=29)$, and a sinus rhythm group, i.e. $R H D+$ sinus rhythm $(n=12)$. Patients with congenital heart disease (CHD) and sinus rhythm who underwent heart surgery served as controls $(n=10)$. Fibrosis was assessed by histological examination, and expression of $a$-actinin-2, TGF- $\beta 1$ and Smad2/phosphorylated Smad2 ( $p$ Smad2) was evaluated by immunohistochemistry, quantitative real-time PCR and Western blotting. In rat atrial fibroblasts treated with TGF- $\beta 1$, the collagen content was measured using hydroxyproline detection, and a-actinin-2 and $\mathrm{p}$-Smad 2 were evaluated by semiquantitative reverse-tran-
\end{abstract}

scription PCR and Western blotting. Results: The histology results revealed a significant increase in atrial fibrosis in AF patients. The collagen content, mRNA and protein expression levels of $a$-actinin- 2 and the components of the TGF- $\beta 1 /$ Smad signaling pathway were significantly gradually increased in the CHD + sinus rhythm, RHD + sinus rhythm and RHD + cAF groups $(p<0.05)$. The mRNA and protein levels of $\alpha$-actinin- 2 and TGF- $\beta 1$ in RHD patients were positively correlated with the collagen volume fraction. A positive correlation between the expression of $\alpha$-actinin- 2 and TGF- $\beta 1$ was also observed. In rat atrial fibroblasts treated with TGF- $\beta 1$, the collagen content was greater than that in the control group ( $p<0.05)$, and the expression levels of $a-$ actinin- 2 and $p$-Smad2 were also upregulated $(p<0.05)$. Conclusions: $a$-Actinin- 2 expression was increased in the atrial tissues of patients with AF secondary to RHD. a-Actinin-2 was upregulated via the TGF- $\beta 1 /$ Smad pathway in atrial fibroblasts, which suggests that it may be involved in TGF- $\beta 1 /$ Smad pathway-induced atrial fibrosis in patients with AF.

(c) 2016 S. Karger AG, Basel

\section{Introduction}

Atrial fibrillation (AF), one of the most common arrhythmias observed in clinical practice, is associated with increased morbidity, particularly due to stroke and heart

\section{KARGER}

E-Mail karger@karger.com

www.karger.com/crd
(C) 2016 S. Karger AG, Basel

$0008-6312 / 16 / 1353-0151 \$ 39.50 / 0$
Hua Xiao, Department of Cardiology

The First Affiliated Hospital of Chongqing Medical University

No. 1 Youyi Road, Yuzhong District

Chongqing 400016 (China)

E-Mail xiaoheart1974@163.com 
failure, and increased mortality [1]. Atrial structural remodeling can lead to atrial dilation and atrial fibrosis in patients with AF [2].

a-Actinin is an essential cytoskeletal protein that plays a primary role in cross-linking actin filaments and is critical for focal adhesion formation and cytoskeletal stability throughout the cell. As a critical component of stress fibers, $a$-actinin- 2 enables cells to withstand shear force and stretch stress $[3,4]$. Atrial structural changes caused by stretching that occur before and during early AF prepare for permanent $\mathrm{AF}$ [5]. In mouse mammary epithelial NMuMG cells, human breast cancer MDA-MB-231 cells and kidney HEK293T cells, overexpressed $\alpha$-actinin is regulated by transforming growth factor- $\beta_{1}$ (TGF- $\beta 1$ ) [6] .

The TGF- $\beta 1 /$ Smad pathway plays a central role in AFrelated cardiac fibrogenesis [7]. Based on this evidence and on our previous study [8], we hypothesize that $\alpha$-actinin-2 plays an important role in the structural remodeling of AF pathogenesis. This study determines whether $\alpha$-actinin-2 exerts its effect on atrial fibrosis through the TGF- $\beta 1 / \mathrm{Smad}$ signaling pathway in patients with AF.

\section{Methods}

\section{Patients and Specimens}

A total of 41 patients (27 females and 14 males; mean age 50.3 \pm 8.2 years) with rheumatic heart disease (RHD) who underwent valve replacement surgery between February and September 2014 at the First Affiliated Hospital of Chongqing Medical University were enrolled in this study. The patients were divided into a sinus rhythm group ( $\mathrm{RHD}+$ sinus rhythm, $\mathrm{n}=12$ ) and a chronic $\mathrm{AF}(\mathrm{AF}$ lasting $\geq 6$ months) group (RHD $+c A F, n=29)$. Patients with congenital heart disease (CHD) and sinus rhythm who underwent heart surgery were included as controls $(\mathrm{CHD}+$ sinus rhythm, $n=10$ ). All surgical electrocardiograms, chest X-rays and echocardiograms were recorded. No patients included in this trial received any type of angiotensin-converting enzyme inhibitor during the 6-month period prior to the study, and all heart functions in patients were New York Heart Association (NYHA) class II-III on admission. Patients with hypertension, coronary artery disease, hyperthyroidism or a malignant tumor and all patients $>70$ years of age were excluded from this study.

Right atrial appendages $(>100 \mathrm{mg})$ were obtained as specimens prior to the establishment of extracorporeal circulation, and were then divided into 2 parts. One part of the tissue was fixed in $4 \%$ paraformaldehyde or $4 \%$ glutaraldehyde for histological analysis or electron microscopy, and the other part was frozen in liquid nitrogen and stored at $-80^{\circ} \mathrm{C}$ for quantitative real-time PCR, reverse transcription PCR and Western blotting.

Our study using human tissue collection abided strictly by the principles outlined in the World Medical Association Declaration of Helsinki. Informed consent was obtained from each patient, and the ethics committee of the First Affiliated Hospital of Chongqing Medical University approved the study.
Histological Staining and Fibrosis Quantification

The specimens were fixed in 4\% paraformaldehyde, embedded in paraffin and cut into 4 - $\mu$ m-thick slices. The slices were deparaffinized with dimethyl benzene and immersed in a series of solutions with a decreasing concentration of alcohol ranging from 100 to $75 \%$. Preparations were stained with hematoxylin and eosin (HE) and Masson's trichrome according to routine procedures. An Olympus BX51 microscope (Tokyo, Japan) was used to observe 5 different fields of each stained slice at $\times 400$ magnification. Collagen fibers in the endocardium were identified. A semiquantitative analysis of atrial appendage collagen fibers was conducted using the ImagePro Plus 6.0 image analysis system. The following formula was used to calculate the collagen volume fraction (CVF): collagen fiber area/total viewed area $\times 100 \%$.

\section{Immunohistochemistry and Electron Microscopy}

An immunohistochemistry kit was obtained from Zhong Shan Golden Bridge Biotechnology Corp. (Beijing, China). Paraffinembedded tissue was cut into 4 - $\mu$ m-thick sections, washed with phosphate-buffered saline (PBS) 3 times for $5 \mathrm{~min}$, and blocked with $1 \%$ goat serum albumin in PBS for $30 \mathrm{~min}$. Each section was then incubated with TGF- $\beta 1$, anti-phospho-Smad 2 and anti- $\alpha$ actinin-2 antibodies (all 1:100 and from Abcam, UK) overnight at $4^{\circ} \mathrm{C}$. The next day, the preparations were washed 3 times with PBS, incubated with peroxidase-conjugated goat anti-rabbit immunoglobulin $\mathrm{G}$ for $20 \mathrm{~min}$, and then washed for $30 \mathrm{~s}$ with diaminobenzidine to detect the peroxidase activity. The specimens were then rinsed with water, counterstained with hematoxylin, and mounted with neutral balsam. Images were captured with an Olympus BX51 microscope (Tokyo, Japan) at $\times 400$ magnification. Electron microscopy images of the atrial appendage were obtained using a $\mathrm{Hi}$ tachi-7500 transmission electron microscope (Tokyo, Japan) at the College of Life Science, Chongqing Medical University.

\section{Quantitative Real-Time PCR}

Total RNA was extracted from the tissue specimens with the RNAiso Plus reagent (Takara, Dalian, China). The RNA was reverse-transcribed into cDNA using a PrimeScript RT reagent kit with gDNA Eraser (Takara, Dalian, China). Quantitative real-time PCR (RT-PCR) was performed using gene-specific primers (table 1) and a SYBR Premix Ex Taq II kit (Takara, Dalian, China), and $\beta$-actin was used as an internal control. The results are expressed as the fold difference for each gene against $\beta$-actin, using the $2^{-\Delta \Delta C T}$ method.

\section{Cell Culture and Treatment}

The Ethics Committee of Chongqing Medical University approved all experimental procedures involving animals. All primary cell collections were performed under sodium pentobarbital anesthesia, and all efforts were made to minimize suffering. The hearts from 2-week-old Sprague-Dawley rats were used. Briefly, we used small scissors and forceps to remove the heart. Following this procedure, the myocardial cells were removed, and purified primary cells were maintained in DMEM-F12 (HyClone, Utah, USA) containing $20 \%$ fetal bovine serum (FBS, Gibco, Calif., USA). DMEM-F12 containing 10\% FBS was used for subculturing the atrial fibroblasts, and the 3rd-5th generation of cells was used for the experiments. Cells were starved for $24 \mathrm{~h}$ and then treated for $24 \mathrm{~h}$ with recombinant human TGF- $\beta 1$ (5 ng/ml; Peprotech, N.J., USA). 


\section{Collagen Content Assay}

The collagen content of the atrial fibroblasts was measured using a hydroxyproline detection kit (Nanjing Jiancheng Bioengineering Institute, Nanjing, China) according to the manufacturer's instructions. The content of collagen per microgram of atrial fibroblasts was calculated by multiplying the hydroxyproline content of the sample by 7.46 .

\section{Semiquantitative Reverse-Transcription PCR for the}

Assessment of $\alpha$-Actinin-2 Expression

RNA was isolated from the atrial fibroblasts using the RNAiso Plus reagent (Takara, Dalian, China). Total RNA $(1 \mu \mathrm{g})$ was used as a reverse-transcription template for the PrimeScript $\mathrm{RT}$ reagent kit (Takara, Dalian, China). Semiquantitative RT-PCR was performed using primers (see table 1 for details) and a Premix Taq kit (Takara, Dalian, China), and $\beta$-actin was used as an internal control. The PCR products were analyzed with Quantity One imaging software (v4.6.3, Bio-Rad, USA).

\section{Western Blotting}

Tissue specimens and atrial fibroblasts washed with PBS were lysed with RIPA lysis buffer containing a 1:100 dilution of protease inhibitor and phosphatase inhibitor (Beyotime Institute of Biotechnology, Shanghai, China). Protein concentrations were measured using a bicinchoninic acid assay kit (Thermo Scientific, Mass., USA). Equal amounts of protein mixtures were subjected to sodium dodecyl sulfate-polyacrylamide gel electrophoresis and blotted onto polyvinylidene difluoride membranes (Millipore, Mass., USA). The membranes were incubated in Tris-buffered saline containing $0.1 \%$ Tween-20 (TBST) with 5\% skimmed milk for $2 \mathrm{~h}$ at $37^{\circ} \mathrm{C}$ and then incubated overnight at $4{ }^{\circ} \mathrm{C}$ with primary antibodies against TGF- $\beta 1$, phospho-Smad 2 and $\alpha$-actinin- 2 (all 1:1,000 and from Abcam). A glyceraldehyde-3-phosphate dehydrogenase (GAPDH) antibody (1:2,000; Immunoway, Calif., USA) was used as an internal control. After 3 washes with TBST, the blot was treated with horseradish peroxidase-conjugated secondary antibodies for $2 \mathrm{~h}$ at $37^{\circ} \mathrm{C}$. After rinsing, the membranes were subjected to enhanced chemiluminescence (Millipore). The ratio of the protein of interest to GAPDH was measured and densitometrically analyzed using Quantity One imaging software v4.6.3.

\section{Statistical Analysis}

Student's t test was used to compare continuous variables between 2 groups, and one-way analysis of variance was used when $\geq 3$ experimental conditions were compared. Student-NewmanKeuls tests were used to determine the differences between groups in a subset of experiments. A linear correlation analysis was used to test the relationships between quantitative parameters. Data for the continuous variables are expressed as means \pm SD. All statistical analyses were performed using SPSS 20.0 (IBM, USA). Statisti$\mathrm{cal}$ significance was assumed at values of $\mathrm{p}<0.05$.

\section{Results}

\section{Clinical Findings}

The patients' clinical characteristics are summarized in table 2. No significant differences were observed between groups for left ventricular ejection fraction, sex or
Table 1. Primer sets for PCR amplification

\begin{tabular}{|c|c|c|}
\hline Gene & \multicolumn{2}{|c|}{ Oligonucleotide primer sequences $\left(5^{\prime}-3^{\prime}\right)$} \\
\hline \multicolumn{3}{|l|}{ Human } \\
\hline \multirow[t]{2}{*}{ TGF- $\beta 1$} & forward: & CAGCAACAATTCCTGGCGATAC \\
\hline & reverse: & GCTAAGGCGAAAGCCCTCAAT \\
\hline \multirow[t]{2}{*}{ SMAD2 } & forward: & GAAACCTTCCATGCATCACAGC \\
\hline & reverse: & CTTCTTGTCATTTCTACCGTGGC \\
\hline \multirow[t]{2}{*}{ ACTN2 } & forward: & GAAGCACAAGCCACCCAAGG \\
\hline & reverse: & CACCAGCAATATCCGACACCA \\
\hline \multirow[t]{2}{*}{$\beta$-Actin } & forward: & CCACGAAACTACCTTCAACTCC \\
\hline & reverse: & GTGATCTCCTTCTGCATCCTGT \\
\hline \multicolumn{3}{|l|}{ Rat } \\
\hline \multirow[t]{2}{*}{ ACTN2 } & forward: & GATAGATTGGGGACGCTCAC \\
\hline & reverse: & GATGGCTAGGATGGACTGC \\
\hline \multirow[t]{2}{*}{$\beta$-Actin } & forward: & AGATGACCCAGATCATGTTTGA \\
\hline & reverse: & TTGGCATAGAGGTCTTTA \\
\hline
\end{tabular}

Table 2. Clinical data of patients

\begin{tabular}{|c|c|c|c|}
\hline & $\begin{array}{l}\mathrm{CHD}+ \\
\text { sinus rhythm }\end{array}$ & $\begin{array}{l}\text { RHD + } \\
\text { sinus rhythm }\end{array}$ & $\mathrm{RHD}+\mathrm{cAF}$ \\
\hline Number of cases & 10 & 12 & 29 \\
\hline Gender, $\mathrm{M} / \mathrm{F}$ & $4 / 6$ & $7 / 5$ & $7 / 22$ \\
\hline Age, years & $45.4 \pm 2.3$ & $48.8 \pm 11.0$ & $50.9 \pm 7.0$ \\
\hline LVEF, \% & $65.4 \pm 4.0$ & $58.2 \pm 8.2$ & $58.4 \pm 7.4$ \\
\hline $\mathrm{LAD}, \mathrm{mm}$ & $26.9 \pm 6.3$ & $39.2 \pm 6.9^{*}$ & $50.2 \pm 6.6^{*, \#}$ \\
\hline LVEDD, mm & $45.8 \pm 5.6$ & $49.3 \pm 6.5$ & $50.1 \pm 3.7$ \\
\hline $\mathrm{RAD}, \mathrm{mm}$ & $36.4 \pm 6.6$ & $37.6 \pm 2.9$ & $39 \pm 6.2$ \\
\hline
\end{tabular}

Data are expressed as means $\pm \mathrm{SD}$, unless indicated otherwise. $\mathrm{LAD}=$ Left atrium dimension; $\mathrm{RAD}=$ right atrium dimension; LVEDD = left ventricular end-diastolic dimension; $\mathrm{LVEF}=$ left ventricular ejection fraction. $* \mathrm{p}<0.05$ (compared with $\mathrm{CHD}+$ sinus rhythm), ${ }^{\#} \mathrm{p}<0.05$ (compared with RHD + sinus rhythm).

age. The left atrial diameter in the RHD + cAF group was significantly larger than that in the RHD + sinus rhythm and CHD + sinus rhythm groups $(\mathrm{p}<0.01)$. The left atrial diameter in the RHD + sinus rhythm group was also significantly larger than that in the CHD + sinus rhythm group $(\mathrm{p}<0.01)$.

\section{Pathological Examination and Quantification of the Fibrotic Area}

$\mathrm{HE}$ and Masson's trichrome staining of the atrial appendage were performed to evaluate atrial fibrosis (fig. 1). $\mathrm{HE}$ staining of the muscle fibers in the RHD + cAF group 


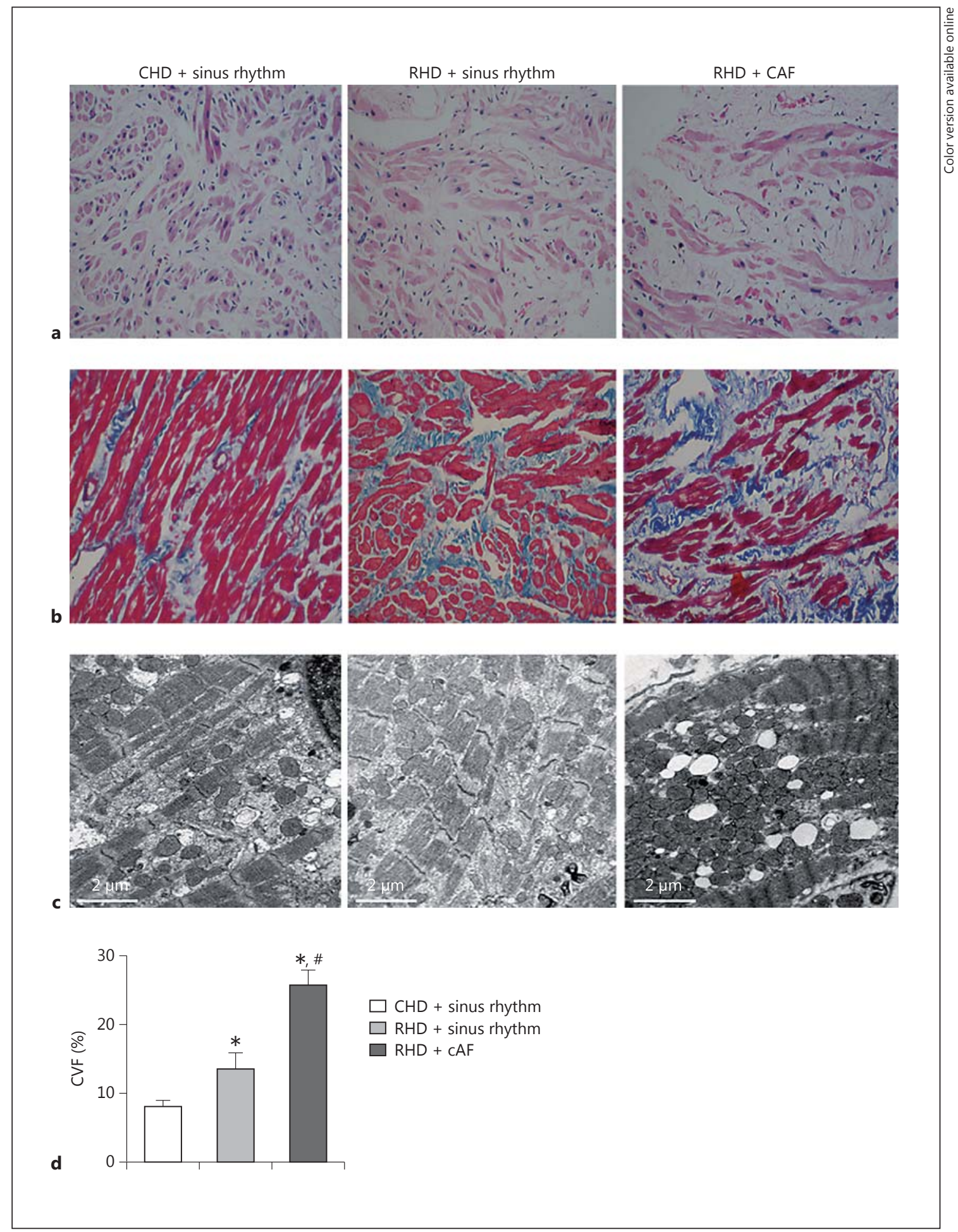

Fig. 1. a HE. $\times 200$. b Masson's trichrome $\times 200$. c Electron microscopy. $\times 10,000$. Disarrayed atrial muscle distribution and myocytolysis were revealed by HE staining and electron microscopy in the RHD + sinus rhythm and $\mathrm{RHD}+\mathrm{cAF}$ groups compared to the CHD + sinus rhythm group. d A significant difference was observed in the CVF among the 3 groups. The values represent the means \pm SD. ${ }^{*} \mathrm{p}<0.05$ (compared with $\mathrm{CHD}+$ sinus rhythm), ${ }^{*} \mathrm{p}<0.05$ (compared with RHD + sinus rhythm). 


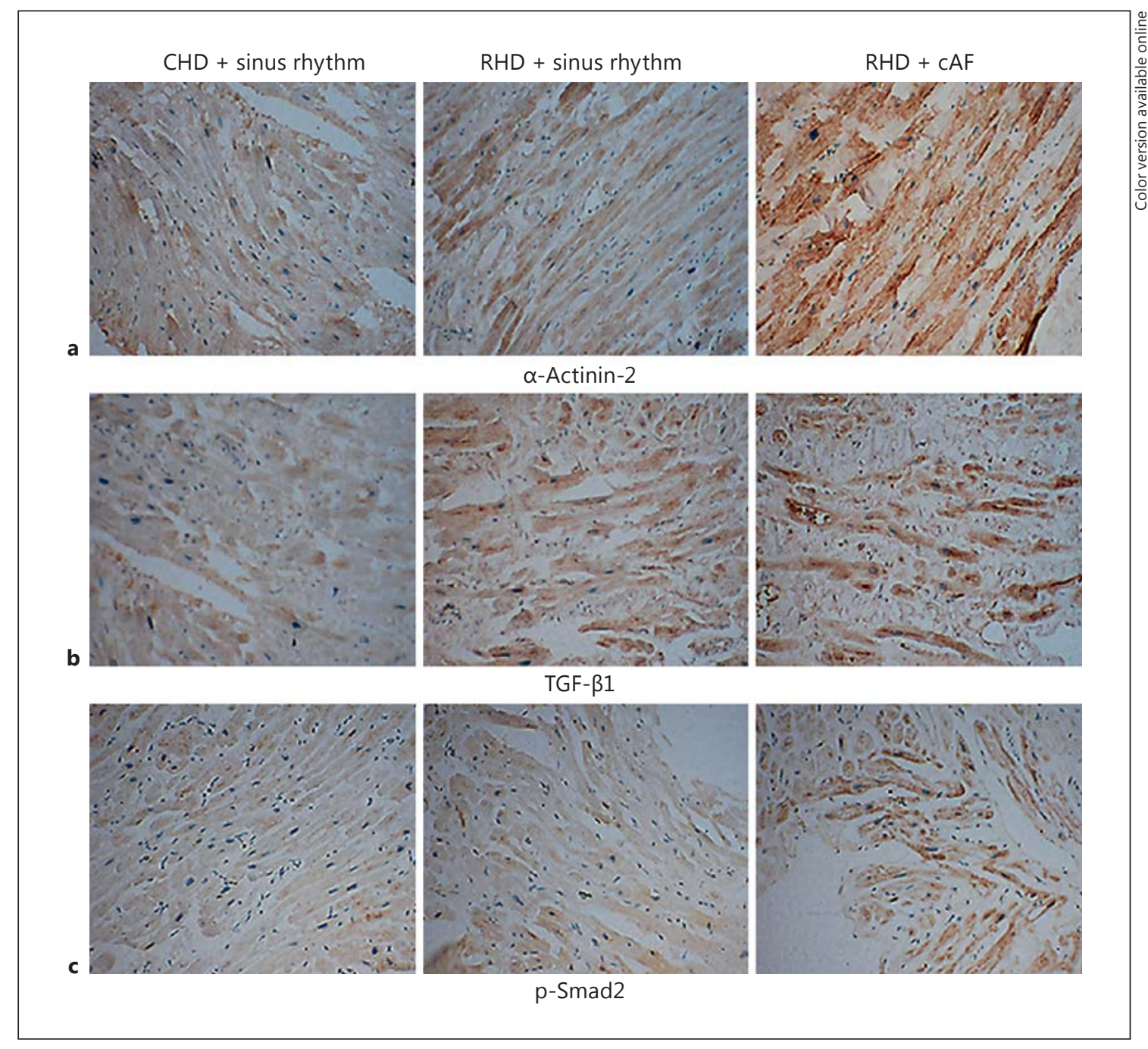

Fig. 2. Immunohistochemical analysis of $\alpha$-actinin-2 (a), TGF- $\beta 1$ (b), and p-Smad2 (c) in the CHD + sinus rhythm, RHD + sinus rhythm and RHD + cAF groups. $\times 200$. Brown- or yellow-stained proteins were distributed mainly throughout the cytoplasm of myocardial cells, and their expression increased gradually.

revealed disordered arrangements and cellular enlargement. Masson's trichrome staining showed blue-stained collagen fibers primarily distributed throughout the myocardial matrix. Collagen fiber expression in the RHD + cAF group was significantly increased compared to that in the RHD + sinus rhythm and CHD + sinus rhythm groups ( $\mathrm{p}<0.05$; fig. 1$)$. Furthermore, collagen fiber expression in the RHD + sinus rhythm group was significantly increased compared to that in the control group ( $\mathrm{p}<0.05$; fig. 1).

\section{Electron Microscopy and Immunohistochemistry}

Neatly arranged, parallel and completed sarcomeres, isometric mitochondria and nontwisting intercalated discs were visible on the images of the control group. Similar characteristics were seen in the RHD + sinus rhythm group. Vague and ruptured sarcomeres of varying lengths, clustered mitochondria and twisting intercalated discs were observed in the RHD + cAF group (fig. 1).

Immunohistochemistry was performed to determine the quantity and location of molecules in the TGF- $\beta 1 /$ Smad signaling pathway and $\alpha$-actinin-2. TGF- $\beta 1, p-$ Smad 2 and $\alpha$-actinin- 2 were expressed in the atrial inter- 
Fig. 3. The mRNA and protein expression levels of $\alpha$-actinin-2 (a), TGF- $\beta 1$ (b) and Smad2/p-Smad2 (c) in the RHD + cAF group were significantly higher than those in the RHD + sinus rhythm and CHD + sinus rhythm groups. The mRNA and protein expression levels of the above molecules in the RHD + sinus rhythm group were significantly higher than those in the $\mathrm{CHD}+$ sinus rhythm group. The values represent the means \pm SD. ${ }^{*} \mathrm{p}<0.05$ (compared with $\mathrm{CHD}+$ sinus rhythm), ${ }^{\#} \mathrm{p}<0.05$ (compared with RHD + sinus rhythm).

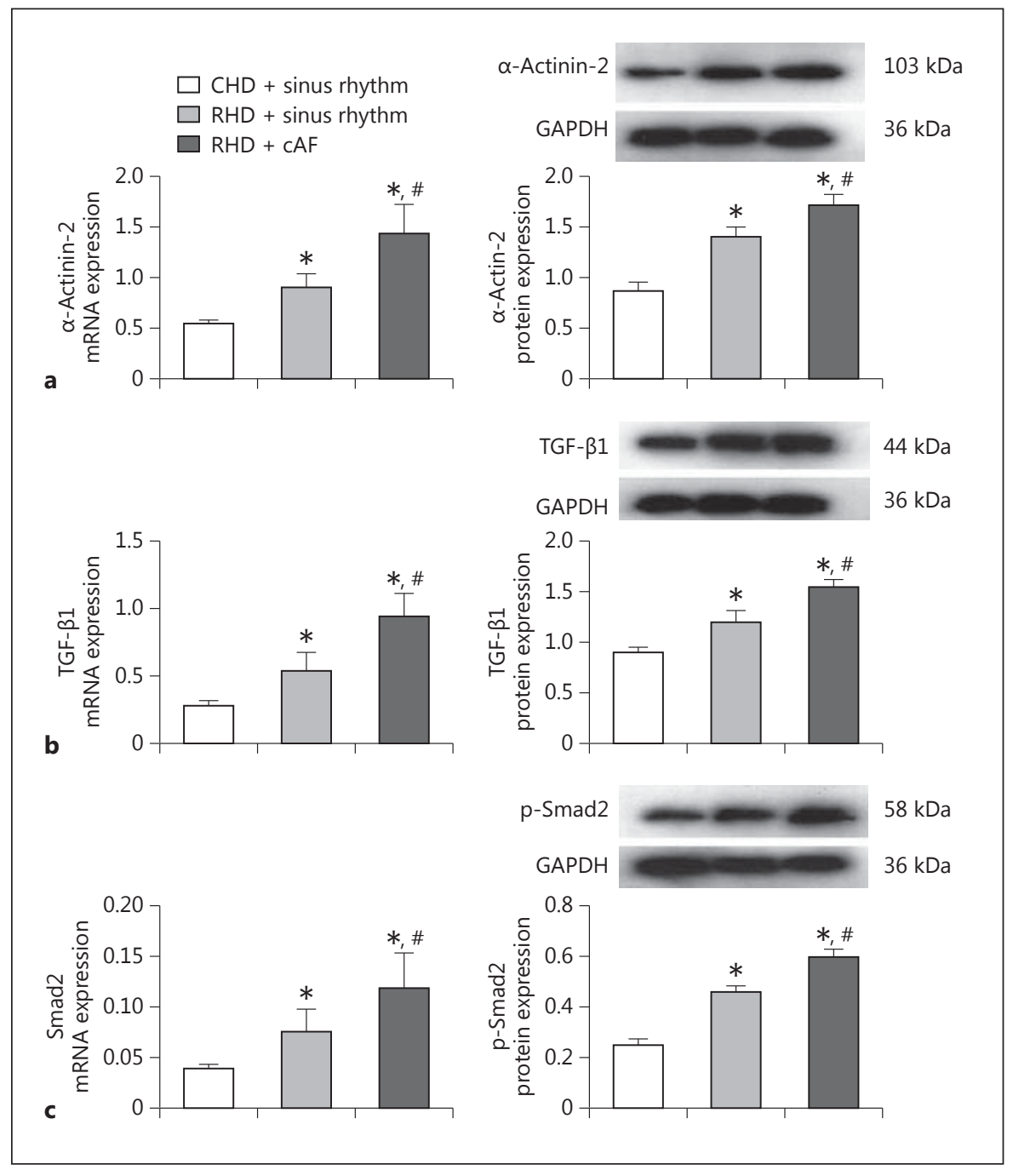

stitium and myocytes in all groups. The expression of the above molecules increased gradually, with reduced expression in the CHD + sinus rhythm group and increased expression in the RHD + sinus rhythm and RHD + cAF groups (fig. 2).

\section{Quantitative Real-Time PCR and Western Blotting in} the Tissues

The mRNA and protein expression levels of $\alpha$-actinin-2, TGF- $\beta 1$ and Smad2/p-Smad2 in the RHD + cAF group were significantly higher than those in the RHD + sinus rhythm and CHD + sinus rhythm groups ( $\mathrm{p}<0.05$; fig. 3 ). Moreover, the mRNA and protein expression levels of the above molecules in the RHD + sinus rhythm group were significantly increased compared to those in the control group ( $\mathrm{p}<0.05$; fig. 3 ).
Collagen Content and Expression of $\alpha$-Actinin-2 and p-Smad 2 in Treated Cells

The collagen content in rat atrial fibroblasts after treatment with TGF- $\beta 1$ was greater than that observed in the control group ( $\mathrm{p}<0.05)$. Additionally, $\alpha$-actinin- 2 and $\mathrm{p}$-Smad2 were upregulated $(\mathrm{p}<0.05)$ in rat atrial fibroblasts after treatment with TGF- $\beta 1$ (fig. 4 ).

\section{Correlation Analysis}

The protein levels of TGF- $\beta 1$ and $\alpha$-actinin- 2 in the RHD patients were positively correlated with the CVF (TGF- $\beta 1$ protein: $r=0.522, p>0.05 ; \alpha$-actinin-2 protein: $r=0.576$, $p>0.05)$. The protein expression of $\alpha$-actinin- 2 was correlated with the TGF- $\beta 1$ level. The correlation between $\mathrm{p}$ Smad 2 and TGF- $\beta 1$ was also significant ( $\alpha$-actinin- 2 protein: $\mathrm{r}=0.913, \mathrm{p}<0.05 ; \mathrm{p}-\mathrm{Smad} 2$ protein: $\mathrm{r}=0.982, \mathrm{p}<0.01$ ). 
Fig. 4. a The collagen content in rat atrial fibroblasts after treatment with TGF- $\beta 1$ was greater than that of the control group $\left({ }^{*} \mathrm{p}<0.05\right)$. Furthermore, the mRNA and protein expression levels of $\alpha$-actinin-2 (b, d) and protein expression level of $\mathrm{p}-\mathrm{Smad} 2$ (c) were upregulated $(\mathrm{p}<0.05)$ in rat atrial fibroblasts after treatment with TGF- $\beta 1$. $\mathrm{M}=$ Marker.

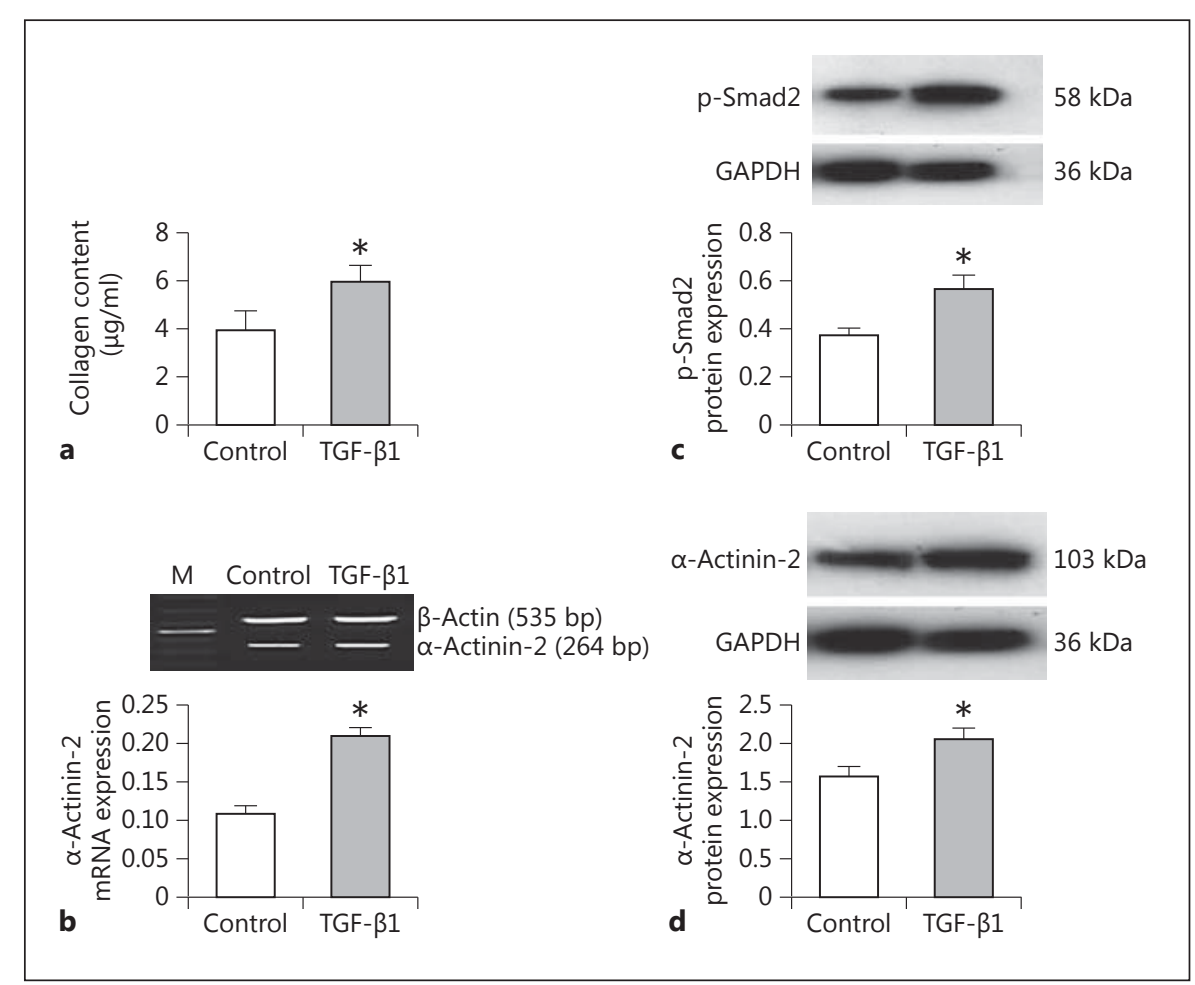

\section{Discussion}

\section{Atrial Structural Remodeling and AF}

AF is the most common arrhythmia, and its prevalence increases with age [9]. AF secondary to rheumatic heart disease is common; it impairs cardiac function and is associated with an increased risk of systemic embolization. Previous studies have shown that atrial remodeling is the basis for the occurrence and maintenance of $\mathrm{AF}$ $[10,11]$. Atrial remodeling consists of structural and electrical remodeling. As a hallmark of atrial structural remodeling, atrial fibrosis has been implicated in tissue biopsies from AF patients and animal models of AF [12]. Atrial fibrosis alters atrial electrical conduction and excitability and provides a substrate for AF maintenance [13]. Atrial fibrosis is characterized by the accumulation of collagen, which replaces degenerating myocytes [14]. In this study, we found that the CVF was significantly increased in patients with $\mathrm{CAF}$, which was consistent with the findings of a previous study [8]. We also found that the CVF was more significantly increased in RHD patients than in CHD patients, which suggests that atrial fibrosis is more serious in the former. This may be one reason why AF secondary to RHD is common in clinical practice. A previous study has shown that TGF- $\beta 1$, which has a profibrotic effect via Smad proteins, is confirmative of AF [15]. It is unknown whether other proteins are involved.

\section{$\alpha$-Actinin-2 Protein and AF}

a-Actinins are cytoskeletal, actin-binding proteins that are members of the spectrin superfamily, comprised of spectrin, dystrophin and their homologs and isoforms [16]. $\alpha$-Actinins are the main components of the Z-disk and account for $<20 \%$ of the Z-disk weight [17]. They play a pivotal role in the assembly of sarcomeres and the regular arrangement of myofilaments [18]. $\alpha$-Actinin-2 is the major cardiac muscle isoform among of the $4 a$-actinins. Cytoskeletal proteins form the sarcomeres, which are the basic contractile units of striated muscle cells [17]. Each sarcomere is bound by the Z-disk [19]. The Z-disk is crucial for maintaining sarcomeric structure and function. The cytoskeleton spatially organizes the contents of the cell, connects the cell physically and biochemically to the external environment, and generates coordinated forces that enable the cell to move and change shape [20]. In recent years, studies have shown that $\alpha$-actinin-2 plays an important role in the pathogenesis of many diseases. It is significantly decreased in individuals with obesity and type 2 diabetes [21]. A genome-wide analysis found that 
mutations in a-actinin-2 cause hypertrophic cardiomyopathy [22]. Exome sequencing has been used to identify a mutation in the $\alpha$-actinin-2 gene in a family associated with idiopathic ventricular fibrillation, left ventricular noncompaction and sudden death [23]. A targeted nextgeneration sequencing approach allowed the identification of a novel $\alpha$-actinin- 2 variant associated with midapical hypertrophic cardiomyopathy and juvenile-onset atrial fibrillation [24]. One study showed remodeling of the sarcomeric cytoskeleton in cardiac ventricular myocytes during heart failure and after cardiac resynchronization therapy [25]. Only a few studies have reported the structural remodeling of the sarcomeric cytoskeleton in AF patients. In our study, compared to the control group, the mRNA and protein expression levels of $\alpha$-actinin-2 were significantly increased in the RHD + sinus rhythm and RHD + cAF groups. We also found that the protein levels of $\alpha$-actinin- 2 in the RHD patients were positively correlated with the CVF. Electron microscopy showed vague and ruptured sarcomeres of varying lengths, clustered mitochondria and twisting intercalated discs in the RHD + cAF group. These results suggest that $\alpha$-actinin-2 may be involved in atrial remodeling in patients with AF.

\section{$\alpha$-Actinin- 2 and the TGF- $\beta 1 /$ Smad Pathway in}

Patients with RHD

TGF- $\beta 1$ plays a critical role in matrix remodeling and enhancing collagen synthesis, both of which induce fibrosis [26]. Transgenic mice overexpressing activated cardiac TGF- $\beta 1$ reportedly developed selective interstitial fibrosis in the atrial myocardium instead of in the ventricular myocardium [27], and were susceptible to AF [28]. TGF- $\beta 1$ exhibits its profibrotic effect primarily through Smad2 and Smad4 [29]. We found that the mRNA and protein expression levels of $\alpha$-actinin-2, TGF- $\beta 1$ and Smad $2 / \mathrm{p}$-Smad 2 were significantly gradually increased in the CHD + sinus rhythm, RHD + sinus rhythm and RHD + cAF groups. These results indicated that the TGF- $\beta 1 /$ Smad pathway plays an important role in $\operatorname{AF}[30,31]$. More importantly, we found that the mRNA and protein expression levels of the above molecules in the RHD + sinus rhythm group were significantly higher than those in the control group. These results implied that $\alpha$-actinin- 2 in patients with RHD is already activated by the TGF- $\beta 1 /$ Smad pathway, even though sinus rhythm is maintained. In patients with RHD + sinus rhythm, the rate of development of AF was increased according to the large left atrium size and mitral stenosis severity [32]. Patients with RHD in sinus rhythm may develop AF during the course of the disease on account of activation of the pathway.

Moreover, an analysis showed that the protein expression of $\alpha$-actinin- 2 was correlated with the TGF- $\beta 1$ level ( $p>$ 0.05 ), possibly due to an inadequate sample amount. This result further indicates that $\alpha$-actinin- 2 may be involved in TGF- $\beta 1 /$ Smad pathway-induced atrial fibrosis in patients with $\mathrm{AF}$.

We further verified the involvement of the TGF- $\beta 1 /$ Smad pathway in vitro. In atrial fibroblasts treated with TGF- $\beta 1$, the collagen content and expression level of $\mathrm{p}$ Smad2 were greater than in the control group. These results are consistent with a previous study $[33,34]$. Furthermore, we found that $a$-actinin-2 expression was greater than that in the control group in atrial fibroblasts treated with TGF- $\beta 1$. This suggests that $\alpha$-actinin- 2 may be the downstream molecule of the TGF- $\beta 1 /$ Smad pathway, and it provides additional evidence for the role of $a$-actinin-2 in fibrotic atrial remodeling. These results support our hypothesis that $\alpha$-actinin- 2 is involved in the structural remodeling of AF pathogenesis.

In summary, we found that $\alpha$-actinin- 2 expression was increased in the atrial tissue in patients with AF secondary to RHD, and that $\alpha$-actinin- 2 was upregulated via the TGF- $\beta 1 /$ Smad pathway in atrial fibroblasts. These findings suggest that $\alpha$-actinin- 2 may be involved in TGF- $\beta$ $1 /$ Smad pathway-induced atrial fibrosis in patients with AF.

\section{Acknowledgements}

This study was supported by the National Natural Science Foundation of China (No. 81300140).

\section{Conflict of Interest}

The authors declare no potential conflicts of interest.

References

Cardiology 2016;135:151-159 DOI: $10.1159 / 000446362$
1 Chugh SS, Havmoeller R, Narayanan K, Singh D, Rienstra M, Benjamin EJ, Gillum RF, Kim YH, McAnulty JH Jr, Zheng ZJ, Forouzanfar MH, Naghavi M, Mensah GA, Ezzati M, Murray CJ: Worldwide epidemiology of atrial fibrillation: a global burden of disease 2010 study. Circulation 2014;129:837-847.

2 Nattel S, Burstein B, Dobrev D: Atrial remodeling and atrial fibrillation: mechanisms and implications. Circ Arrhythm Electrophysiol 2008;1:62-73.

3 Shams H, Golji J, Mofrad MR: A molecular trajectory of alpha-actinin activation. Biophys J 2012;103:2050-2059. 
4 Gautel M: The sarcomeric cytoskeleton: who picks up the strain? Curr Opin Cell Biol 2011; 23:39-46.

5 De Jong AM, Maass AH, Oberdorf-Maass SU, Van Veldhuisen DJ, Van Gilst WH, Van Gelder IC: Mechanisms of atrial structural changes caused by stretch occurring before and during early atrial fibrillation. Cardiovasc Res 2010;89:754-765.

6 Bakin AV, Safina A, Rinehart C, Daroqui C, Darbary H, Helfman DM: A critical role of tropomyosins inTGF-beta regulation of the actin cytoskeleton and cell motility in epithelial cells. Mol Biol Cell 2004;15:4682-4694.

7 Gramley F, Lorenzen J, Koellensperger E, Kettering K, Weiss C, Munzel T: Atrial fibrosis and atrial fibrillation: the role of the TGF- $\beta 1$ signaling pathway. Int J Cardiol 2010; 143:405-413.

8 Xiao H, Lei H, Qin S, Ma K, Wang X: TGFbetal expression and atrial myocardium fibrosis increase in atrial fibrillation secondary to rheumatic heart disease. Clin Cardiol 2010; 33:149-156.

9 Fuster V, Ryden LE, Cannom DS, et al: 2011 ACCF/AHA/HRS focused updates incorporated into the ACC/AHA/ESC 2006 guidelines for the management of patients with atrial fibrillation: a report of the American College of Cardiology Foundation/American Heart Association Task Force on practice guidelines. Circulation 2011;123:e269-e367.

10 Maurits A, Jannie A, Ulrich S: Electrical, contractile and structural remodeling during atrial fibrillation. Cardiovasc Res 2002;54:230246.

11 Iwasaki YK, Nishida K, Kato T, Nattel S: Atrial fibrillation pathophysiology: implications for management. Circulation 2011;124:22642274.

12 Everett TH, Olgin JE: Atrial fibrosis and the mechanisms of atrial fibrillation. Heart Rhythm 2007;4:S24-S27.

13 Tan AY, Zimetbaum P: Atrial fibrillation and atrial fibrosis. J Cardiovasc Pharmacol 2011; 57:625-629.

14 Burstein B, Nattel S: Atrial fibrosis: mechanisms and clinical relevance in atrial fibrillation. J Am Coll Cardiol 2008;51:802-809.

15 Khan R, Sheppard R: Fibrosis in heart disease: understanding the role of transforming growth factor-beta in cardiomyopathy, valvu- lar disease and arrhythmia. Immunology 2006;118:10-24.

16 Sjoblom B, Salmazo A, Djinovic-Carugo K: Alpha-actinin structure and regulation. Cell Mol Life Sci 2008;65:2688-2701.

17 Frank D, Kuhn C, Katus HA, Frey N: The sarcomeric z-disc: a nodal point in signalling and disease. J Mol Med (Berl) 2006;84:446-468.

18 Wang J, Shaner N, Mittal B, Zhou Q, Chen J, Sanger JM, Sanger JW: Dynamics of z-band based proteins in developing skeletal muscle cells. Cell Motil Cytoskeleton 2005;61:34-48.

19 Crawford GL, Horowits R: Scaffolds and chap erones in myofibril assembly: putting the striations in striated muscle. Biophys Rev 2011;3: 25-32.

20 Fletcher DA, Mullins RD: Cell mechanics and the cytoskeleton. Nature 2010;463:485-492.

21 Hwang H, Bowen BP, Lefort N, Flynn CR, De Filippis EA, Roberts C, Smoke CC, Meyer C, Hojlund K, Yi Z, Mandarino LJ: Proteomics analysis of human skeletal muscle reveals novel abnormalities in obesity and type 2 diabetes. Diabetes 2010;59:33-42.

22 Chiu C, Bagnall RD, Ingles J, Yeates L, Kennerson M, Donald JA, Jormakka M, Lind JM, Semsarian C: Mutations in alpha-actinin-2 cause hypertrophic cardiomyopathy: a genome-wide analysis. J Am Coll Cardiol 2010; 55:1127-1135.

23 Bagnall RD, Molloy LK, Kalman JM, Semsarian C: Exome sequencing identifies a mutation in the ACTN2 gene in a family with idiopathic ventricular fibrillation, left ventricular noncompaction, and sudden death. BMC Med Genet 2014;15:99.

24 Girolami F, Iascone M, Tomberli B, Bardi S, Benelli M, Marseglia G, Pescucci C, Pezzoli L, Sana ME, Basso C, Marziliano N, Merlini PA, Fornaro A, Cecchi F, Torricelli F, Olivotto I: Novel alpha-actinin 2 variant associated with familial hypertrophic cardiomyopathy and juvenile atrial arrhythmias: a massively parallel sequencing study. Circ Cardiovasc Genet 2014;7:741-750.

25 Lichter JG, Carruth E, Mitchell C, Barth AS, Aiba T, Kass DA, Tomaselli GF, Bridge JH, Sachse FB: Remodeling of the sarcomeric cytoskeleton in cardiac ventricular myocytes during heart failure and after cardiac resynchronization therapy. J Mol Cell Cardiol 2014;72:186-195.
26 Lijnen P, Petrov V: Transforming growth factor-beta 1-induced collagen production in cultures of cardiac fibroblasts is the result of the appearance of myofibroblasts. Methods Find Exp Clin Pharmacol 2002;24:333-344.

27 Nakajima H, Nakajima HO, Salcher O, Dittiè AS, Dembowsky K, Jing S, Field LJ: Atrial but not ventricular fibrosis in mice expressing a mutant transforming growth factor-beta(1) transgene in the heart. Circ Res 2000;86:571579.

28 Sander V, Toshiaki S, Thomas E, Engle SK, Dan O, Michael RVDL, Nakajima HO, Hidehiro N, Field LJ, Olgin JE: Increased vulnerability to atrial fibrillation in transgenic mice with selective atrial fibrosis caused by overexpression of TGF-beta1. Circ Res 2004;94: 1458-1465

29 Polyakova V, Miyagawa S, Szalay Z, Risteli J, Kostin S: Atrial extracellular matrix remodelling in patients with atrial fibrillation. J Cell Mol Med 2008;12:189-208.

30 Rahmutula D, Marcus GM, Wilson EE, Ding $\mathrm{CH}$, Xiao Y, Paquet AC, Barbeau R, Barczak AJ, Erle DJ, Olgin JE: Molecular basis of selective atrial fibrosis due to overexpression of transforming growth factor-betal. Cardiovasc Res 2013;99:769-779.

31 Zhang D, Liu X, Chen X, Gu J, Li F, Zhang W, Zheng Y: Role of the MAPKs/TGF- $\beta 1 /$ TRAF6 signaling pathway in atrial fibrosis of patients with chronic atrial fibrillation and rheumatic mitral valve disease. Cardiology 2014;129: 216-223.

32 Kim HJ, Cho GY, Kim YJ, Kim HK, Lee SP, Kim HL, Park JJ, Yoon YE, Zo JH, Sohn DW: Development of atrial fibrillation in patients with rheumatic mitral valve disease in sinus rhythm. Int J Cardiovasc Imaging 2015;31: 735-742.

33 Yeh YH, Kuo CT, Chang GJ, Qi XY, Nattel S, Chen WJ: Nicotinamide adenine dinucleotide phosphate oxidase 4 mediates the differential responsiveness of atrial versus ventricular fibroblasts to transforming growth factor-beta. Circ Arrhythm Electrophysiol 2013;6:790-798.

34 Zhang $\mathrm{P}$, Wang W, Wang X, Wang X, Song Y, Zhang J, Zhao H: Focal adhesion kinase mediates atrial fibrosis via the Akt/S6k signaling pathway in chronic atrial fibrillation patients with rheumatic mitral valve disease. Int J Cardiol 2013;168:3200-3207.
Increased $\alpha$-Actinin-2 Expression in Patients with AF Related to RHD
Cardiology 2016;135:151-159 DOI: $10.1159 / 000446362$ 